Rapport - Société canadienne d'histoire de l'Église catholique

\title{
Mère Gamelin et le Service Social
}

\section{Madeleine-Durand}

Volume 28, 1961

URI : https://id.erudit.org/iderudit/1007373ar

DOI : https://doi.org/10.7202/1007373ar

Aller au sommaire du numéro

Éditeur(s)

La Société canadienne d'histoire de l'Église catholique

ISSN

0318-6148 (imprimé)

1927-7075 (numérique)

Découvrir la revue

Citer cet article

Madeleine-Durand (1961). Mère Gamelin et le Service Social. Rapport - Société canadienne d'histoire de l'Église catholique, 28, 11-18.

https://doi.org/10.7202/1007373ar

Tous droits réservés @ La Société canadienne d'histoire de l'Église catholique, 1963
Ce document est protégé par la loi sur le droit d'auteur. L’utilisation des services d'Érudit (y compris la reproduction) est assujettie à sa politique d'utilisation que vous pouvez consulter en ligne.

https://apropos.erudit.org/fr/usagers/politique-dutilisation/ 


\section{Mère Gamelin et le Service Social}

En venant vous entretenir de « Mère Gamelin et le Service Social », je n'ai pas l'intention de présenter une conférence savante, fortement charpentée, à base de documents et étayée de nombreuses références, ce que, d'ailleurs, je n'aurais pu faire. Je veux, tout simplement, souligner un aspect de l'activité charitable de notre vénérée Mère Fondatrice et lui offrir, en même temps, un filial hommage d'admiration et de gratitude.

Ce ne sera une surprise pour personne, nous semble-t-il, que d'entendre une Sour de la Providence parler de la fondatrice de son Institut et du sceau qu'elle a imprimé à la charité dans le Montréal de la dernière moitié du $\mathrm{XIX}^{\bullet}$ siècle.

Ce $\mathrm{XIX}^{\bullet}$ siècle venait à peine de naître que Marie-Emilie-Eugène Tavernier ouvrait les yeux à la lumière. Il est une ironie des noms comme il en est une des choses : la famille dans laquelle l'enfant vient prendre place porte le nom de « Tavernier dit Sanspitié ». Son grandpère, Julien Tavernier, venu de Picardie à Montréal, était sergent dans la Compagnie du chevalier de Lacorne. Il tombait glorieusement au champ d'honneur en juillet 1756, dans les environs du lac Champlain, laissant une veuve et quatre enfants dont le plus jeune, Antoine, fut le père de notre Fondatrice.

Dès ses plus jeunes années, la petite semble vouloir faire mentir le nom de "Sanspitié " accolé à celui de "Tavernier ". La précocité de sa compassion envers les pauvres fait l'étonnement de son entourage. Sa maman, heureuse de constater les belles dispositions de sa fillette, se plaît à faire passer par ses mains enfantines, l'aumône destinée au mendiant qui la sollicite pour l'amour du bon Dieu.

Or, un matin, Emilie voit venir dans le chemin montant qui conduit à la maison paternelle, un vieillard qui s'avance péniblement, appuyé sur son bâton. Elle se hâte à sa rencontre avec son petit panier plein de provisions qu'elle jette dans la grande besace que le mendiant ouvre devant elle. En voyant sa légère offrande s'abîmer dans le fond du sac, elle se met à pleurer et revient vers sa maman : "Maman, maman, le sac n'est pas plein ! . Celle-ci essaie de lui faire comprendre, pour la consoler, que le pauvre commence sa tournée quotidienne et que, à son retour, le soir, il aura suffisamment ramassé pour subvenir aux besoins de sa famille. Mais, pour son cœur compatissant, la besace du pauvre lui paraît déjà, et lui paraîtra toujours, trop grande, jusqu'à ce qu'elle lui ait donné ses biens, sa santé, sa vie, tous les trésors de son esprit et de son cœur.

A celle que le Seigneur destinait à fonder une communauté où toutes les misères humaines trouveraient refuge, les épreuves ne furent pas ménagées. La petite n'a pas encore ses quatre ans qu'elle perd sa 
mère. Plus tard, elle avait alors dix-huit ans, son frère François, devenu veuf, lui confie la tenue de sa maison. Ce rôle de ménagère lui plaît d'autant plus qu'Emilie trouve, dans ce frère aîné, un ami des pauvres qui lui permet d'assister autant de malheureux qu'il s'en présenterait chez lui. "Bon sang ne peut mentir », dit le proverbe. D'une génération à l'autre, les Tavernier et leurs parents ont toujours fait preuve d'une tendre charité envers les déshérités de la vie. François, aussi sensible d'âme que bouillant de caractère, voit avec plaisir sa clientèle de miséreux doubler, et même tripler, grâce à sa jeune sœur.

Dans une petite pièce attenant à la cuisine et que mademoiselle Tavernier appelle gentiment son cabinet particulier, elle aménage une salle à manger à l'usage exclusif de ses amis de choix. C'est là que les pauvres s'asseoient autour d'une grande table que l'aimable jeune fille nomme la table $d u$ roi. Que cette appellation prouve bien le sens chrétien de celle qui sert elle-même, avec une respectueuse tendresse, les préférés du Seigneur! Il nous est doux de croire que la prière reconnaissante de ces malheureux lui aura obtenu une large part des grâces de choix qui l'ont conduite jusqu'à la fondation d'un Institut particulièrement consacré aux œuvres de charité.

Mariée à 23 ans, mademoiselle Tavernier trouve en son époux, M. Jean-Baptiste Gamelin, un cœur porté, comme le sien, à la pitié envers les malheureux. Il laisse donc à sa jeune épouse toute liberté de leur venir en aide, d'autant plus que la modeste aisance dont il jouit lui permet de répondre aux exigences de leur commun amour des pauvres.

Le bonheur, tous en font plus ou moins l'expérience, n'est pas de la terre. Après quatre ans à peine d'une vie conjugale très heureuse madame Gamelin voit mourir cet époux qui l'a entourée d'une tendresse généreuse et discrète. Parmi les biens dont il la fait héritière, il est un legs pour le moins surprenant : c'est celui d'un idiot qui, dans une circonstance périlleuse, a sauvé par ses cris, la vie de M. Gamelin. Celui-ci, en retour, s'est chargé, bien avant son mariage, de pourvoir aux besoins de ce pauvre être et de sa mère. Voyant sa fin approcher, M. Gamelin dit à sa femme : "Prenez soin de lui en souvenir de moi et de mon amour. "Et Dieu sait avec quelle délicate exactitude la jeune veuve a exécuté cette dernière volonté, jusqu'à la mort du pauvre Dodais. Dans notre communauté, ce Dodais - c'est le seul nom que nous lui connaissons - est regardé comme la cellule initiale de l'œuvre qui s'accomplit, depuis près de cent ans, à l'immense hôpital Saint-Jean-de-Dieu et à celui du Mont-Providence où les - Dodais » se chiffrent par centaines.

Un dernier enfant restait à madame Gamelin, les deux autres lui ayant été ravis presque dès le berceau. Dix mois après le décès de son mari, ce petit être sur lequel s'étaient reportées toutes ses affections, quittait aussi la terre. Ce qu'elle éprouva d'affliction à la mort de cet enfant bien-aimé, elle-même nous le dit dans ses notes de retraite de 1850 , moins d'un an avant sa mort. Elle nous apprend en même temps comment elle parvint alors à se résigner à la volonté de Dieu : "Dans cette même année, M. Saint-Pierre (prêtre de Saint-Sulpice, son directeur spirituel) me fit présent d'une image de Notre-Dame-des-Sept-Douleurs 
et tous les jours j'allais prier au pied de cette image. Je demandais à la Sainte Vierge du courage pour supporter à son exemple les croix et les sacrifices que le bon Dieu m'envoyait dans le monde. Le plus grand, dans ce temps-là, était la perte d'un époux et d'un enfant chéris que je pleurais tous les jours. J'avais le cœur percé d'un glaive de douleur et je ne trouvais d'autre consolation que celle de méditer sur les douleurs de ma Mère auprès de cette gravure. *

Quelques semaines après la mort de son mari, le 18 décembre 1827, quelques dames de la première société de Montréal, avec l'approbation de Mgr Lartigue et sous la direction des MM. de Saint-Sulpice, fondent l'Association des Dames de la Charité, dont un comité est particulièrement préposé à la visite des pauvres. Disons en passant que l'hiver 1827-1828 s'annonçant très rigoureux, surtout pour les pauvres, les citoyens de Montréal s'entendirent, dès l'automne, afin d'aviser aux moyens de prévenir les misères ou de les soulager en temps opportun. L'Association des Dames de la Charité compte parmi les moyens les plus efficaces pour venir en aide aux nécessiteux.

Madame Gamelin fait partie du comité de la visite des pauvres à domicile. N'est-il pas merveilleux de constater comment la jeune veuve est conduite, comme par la main, vers ses destins providentiels ?

Libre de toute charge familiale après la mort de son dernier enfant, madame Gamelin peut se donner entièrement à une tâche qui répond si bien aux attraits de son grand et noble cœur.

La visite des pauvres à domicile la met en contact avec certaines misères difficiles à soulager. La détresse des femmes âgées et pauvres qui languissent dans l'indigence et l'isolement, déchire le cœur de la sympathique visiteuse. C'est alors qu'elle croit de son devoir d'adoucir, même aux dépens de son repos, le sort de ces femmes que la décrépitude de l'âge et la pauvreté exposent à mourir dans un complet délaissement. Avec l'encouragement de M. Saint-Pierre et l'appui de M. Claude Fay, curé de Notre-Dame - la seule paroisse du Montréal de 1828 - qui s'empresse de mettre à sa disposition le rez-de-chaussée d'une petite école paroissiale sise à l'angle sud-ouest des rues SainteCatherine et Saint-Laurent, à l'endroit où est situé, de nos jours, un des grands magasins connus sous le nom de cinq-dix-quinze, madame Gamelin ne tarde pas à installer quelques pauvres vieilles dans ce premier refuge et exerce envers elles toutes les formes de service social.

Le petit hospice est vite encombré. Afin de remédier au défaut d'espace, madame Gamelin loue, de son frère Julien, une maison située rue Saint-Philippe (aujourd'hui rue Benoît), où elle peut s'installer elle-même à côté de ses protégées et intervenir pour rétablir l'ordre quand les différends s'élèvent, car il n'est pas toujours facile de faire régner l'entente entre des personnes de cet âge qui ont toujours vécu à leur guise. La charitable dame se rend souvent auprès d'elles pour présider à leurs prières, leur enseigner le catéchisme et les mettre d'accord.

Ce nouveau refuge constitue, pour les ressources de madame Gamelin, une lourde charge. Que de fois elle se demande si elle n'a pas trop 
présumé de ses moyens et tenté la Providence en s'aventurant dans une entreprise dont les lendemains demeurent aussi incertains. La prière des pauvres, sur laquelle madame Gamelin compte beaucoup, reste son grand recours. Dans ses angoisses, elle réunit ses chères vieilles et, après avoir prié, chante avec elles son cantique favori : 0 Douce Providence. Ce cantique est conservé dans l'Institut comme un bien de famille et le chant de ralliement des filles de Mère Gamelin.

En 1832, le choléra fait rage à Montréal. Madame Gamelin est au nomhre des charitahles samaritaines chargées de visiter les malades atteints de l'épidémie. Malgré la peur que lui inspire cette terrible maladie, elle se donne à sa tâche avec son zèle accoutumé. Que de fatigues, que de privations même elle s'impose pour secourir les familles éprouvées. Un jour, après avoir reçu le dernier soupir d'une pauvre femme dont le mari vient de mourir et qui laisse six enfants dans la misère, la bonne dame amène les six orphelins à son hospice à la grande joie de ses vieilles qui se disputent le droit de les dorloter.

L'espace manque bientôt à ce deuxième refuge et son loyer est devenu une lourde charge pour le budget de madame Gamelin. Selon son habitude, elle implore le secours de la bonne Providence, lui demandant une maison plus appropriée à son œuvre. Sa confiance ne sera pas trompée. Monsieur Olivier Berthelet, riche financier de Montréal, venait d'acquérir une propriété qui ferait aujourd'hui l'angle sud-ouest des rues Sainte-Catherine et Saint-Christophe. Ce charitable monsieur, s'étant rendu à l'invitation de visiter le petit hospice de madame Gamelin, y est accueilli avec amabilité par les bonnes vieilles qui se chargent volontiers des frais de la conversation. L'une d'elles, douée d'un aplomb remarquable et d'une plus grande facilité de parole, s'avise - ou peut-être est-elle avisée - de dire au sympathique visiteur : a Mais, monsieur, vous qui avez beaucoup de maisons, vous pourriez bien nous en donner une. " Lorsque le bon M. Berthelet s'elloigne de l'humble refuge, il possède une maison de moins en ce monde, mais un titre de plus aux récompenses éternelles.

Le 13 mai 1836, les vingt-quatre vieilles du refuge sont transférées à la Maison jaune qui deviendra, par la suite, le berceau de la communauté des Sœurs de la Providence. C'est là que, le 25 mars 1843, aura lieu la première prise d'habit, événement considéré comme l'acte de naissance de l'Institut.

Madame Gamelin apporte aussi son concours à une autre cuvre, celle de l'Asile des Filles repenties dont, au dire de ses contemporaines, les intérêts lui tenaient à cœur.

Il est un genre de service social que madame Gamelin a, pour ainsi dire, inauguré, à une époque dont le souvenir ne manque jamais d'émouvoir la fibre patriotique dans les cœurs. C'était l'heure où nos ancêtres luttaient avec désespoir pour la reconnaissance de leur droit à vivre au grand soleil d'un pays qui restait toujours le leur, malgré la conquête.

Plusieurs prisonniers politiques étaient enfermés dans la prison, nouvelle alors, ancienne pour nous, du Pied-du-Courant. Bien qu'un 
bon nombre de ses parents fussent parmi les patriotes compromis, madame Gamelin visitait la prison en toute liberté. Jamais les autorités ne parurent douter de sa prudence et de sa discrétion. Un jour, cependant, elle reçoit la visite d'un fonctionnaire qui lui demande si elle cache des armes dans sa maison. "Mais oui, et les voici », répond-elle vivement en ouvrant la porte d'une salle remplie de vieilles.

Madame Gamelin profitait amplement de cette liberté et s'entremettait volontiers pour favoriser les communications entre les prisonniers et leurs parents. C'est par elle que le notaire Cardinal, dont le procès s'instruisait, faisait parvenir à sa femme, le 13 décembre 1838, une lettre dont nous extrayons les lignes suivantes : " La bonne madame (madame Gamelin) m'a mis en état de t'envoyer aujourd'hui une lettre que je t'ai écrite depuis plusieurs jours et j'espère que demain elle se chargera de celle-ci ... ne crains pas de me compromettre... Cette dame est si bonne, si compatissante, si vertueuse qu'elle ne refusera aucun service qui soit en son pouvoir. Elle paraît s'intéresser beaucoup pour nous...»

Le lendemain du jour où le notaire Cardinal écrivait cette lettre, plusieurs insurgés étaient condamnés à mort. Son nom ouvrait la douloureuse liste; celui de Joseph Duquette, étudiant en droit, figurait ensuite. Tous deux se montraient courageux et résignés, mais en songeant à leurs familles, ils tombaient parfois dans un profond abattement. Cardinal était marié et père de plusieurs enfants; Duquette était l'unique soutien de sa mère, veuve, et de ses trois jeunes sœurs. Madame Gamelin les visitait assidûment. Tout en gardant le premier rang à la charité, elle témoignait ainsi d'un patriotisme éclairé et magnanime.

Au matin du 20 décembre, veille de l'exécution, M. Cardinal lui confiait une lettre pour sa femme. Dans la soirée, il lui écrivait de nouveau et longuement, n'espérant plus sa visite, ni celle d'aucun de ses parents. Il comptait sans l'ingénieuse sympathie de madame Gamelin, car c'est elle, sans doute, qui amenait à la prison, dans la soirée du 20 décembre, les plus proches parents de Cardinal et de Duquette. Madame Marion (Marguerite Cardinal) racontait plus tard à nos sœurs qu'elle avait six ans lorsque son malheureux père fut exécuté. Laissonslui la parole :

- Le 20 décembre, ma mère, ma petite sœur Charlotte et moi, ainsi que madame Duquette et mon grand-père, Bernard Saint-Germain, allions voir mon père et le jeune Duquette à la prison, au Pied-duCourant, où ils étaient détenus depuis un mois. Madame Gamelin, la future fondatrice des Sœurs de la Providence, nous accompagnait. Il était minuit quand nous arrivâmes à la prison. On nous conduisit dans une grande salle... Je vis mon père, deux oncles et d'autres prisonniers politiques. Je ne savais pas que mon père devait mourir le lendemain... Je me rappelle qu'il consolait ma mère et que madame Duquette sanglotait en baisant les mains de son fils. L'entrevue dura une heure. Lorsque nous partîmes, ma mère rapportait une lettre que mon père avait écrite, désespérant de nous voir avant sa mort... Le lendemain, l'exécution de mon père avait lieu à neuf heures et celle du jeune Duquette à neuf heures et demie.» 
Lorsqu'elle vint demeurer à Montréal, madame Cardinal, ainsi que ses enfants, fut l'objet des sympathies de madame Gamelin. « Je me rappelle, dit encore madame Marion, que ma mère, mes sœurs et moi nous allions parfois dîner chez madame Gamelin à sa demeure, rue Sainte-Catherine. »

Dès les premiers jours de janvier 1839, plusieurs autres patriotes furent condamnés. Le tribunal qui sévissait ainsi contre les malheureux prisonniers politiques ne tolérait aucune communication entre les condamnés à mort et leurs parents ou amis, excenté la veille de l'exécution. Madame Jacques Longtin, de Saint-Constant, venue pour visiter son mari condamné le 10 janvier, ne put en obtenir la permission. Elle amenait avec elle sa fille, Sophie, âgée de treize ans. Dans sa détresse, la pauvre femme a recours à madame Gamelin. Celle-ci, afin de la consoler autant qu'elle peut, met un panier au bras de la petite, se charge elle-même de différents objets et s'achemine vers la prison avec sa mignonne compagne. Obligée de traverser la cour entre deux rangées de soldats, l'enfant tremble de tous ses membres; son charitable guide la rassure et la remet à son père qui n'en peut croire ses yeux. Heureuse de ce bonheur dont elle a été l'instrument, madame Gamelin laisse seuls la petite et son père et s'en va distribuer provisions et messages aux autres prisonniers.

Cette petite Sophie Longtin, devenue Sœur de la Providence sous le nom de Sœur Jean-Baptiste, a raconté maintes fois cet épisode de son enfance. Elle est décédée le 13 août 1914, à notre hôpital Saint-Eusèbe, Joliette, dans sa quatre-vingt-dixième année après avoir passé soixanteneuf ans dans l'exercice des œuvres de charité les plus méritoires. Il nous est bien permis de croire que l'attitude toute de bonté et de charité de madame Gamelin envers la famille Longtin contribua, dans une large mesure, à sa vocation de Servante des Pauvres.

La dernière victime des événements qui, depuis 1837, endeuillaient tant de familles canadiennes-françaises, fut le notaire François-MarieThomas-Chevalier de Lorimier. Madame Gamelin, amie de la famille de Lorimier, partagea sa douloureuse affliction. Madame de Lorimier ainsi que ses filles, Léopoldine et Stéphanie, passa la dernière partie de sa vie à L'Assomption. Les Sœurs de la Providence la visitaient souvent et se plaisaient à l'entendre évoquer le souvenir de leur Fondatrice et de son infatigable charité. Madame de Lorimier était âgée de 79 ans lorsqu'elle décéda à L'Assomption, le 6 décembre 1891. Sa fille, Léopoldine mourait le 12 mars 1898 , à l'âge de 65 ans. Sa sœur, Stéphanie, demeurée seule, se retira à notre Hospice Notre-Dame, L'Assomption, où elle décédait à son tour, le 5 mars 1904 .

Plusieurs autres patriotes, dont Jacques Longtin et son fils, Moïse, furent déportés en Australie. L'un d'eux, François-Xavier Prieur, dans ses « Mémoires d'un Prisonnier politique », écrit en parlant de madame Gamelin : "C'était une magnifique femme au langage sympathique et aux manières distinguées. Sa charité était inépuisable. " Ces simples paroles, dans leur brièveté, en disent long sur la réputation de bienfaisance de madame Gamelin. 
Nous pourrions encore citer ce fragment d'une lettre écrite par M. F.-M. LePailleur à sa femme, durant son exil et publiée le 11 décembre 1840 dans L'Aurore des Canadas: "N'oublie pas non plus madame Gamelin et madame Gauvin et dis-leur que, toute ma vie, je me rappellerai leurs bons soins et les services qu'elles m'ont rendus. ”

Madame Gamelin qu'on avait surnommée l'Ange des prisonniers politiques, s'intéressa toujours au sort des malheureux exilés et continua ses attentions à leurs familles. Son dévouement aux infortunées victimes de ces tristes années 1837-1838 lui valut non seulement l'admiration du public, mais lui attira encore d'inappréciables et continuels encouragements et devint une source de bénédictions pour son petit hospice.

Lorsque Mgr Bourget, déçu par l'impossibilité où se trouvèrent les Filles de la Charité de France de venir prendre possession de l'Asile de la Providence, se décida à fonder une communauté canadienne sur le modèle des Sœurs de Saint-Vincent-de-Paul, madame Gamelin, sa courageuse collaboratrice, prenait rang parmi les sept premières Sœurs de la Providence et était nommée supérieure du nouvel Institut dont la date de naissance, comme nous l'avons dit, s'inscrit au 25 mars 1843.

Dès la fin de cette même année 1843 , les novices sont initiées au service social, appellation encore inconnue chez nous, mais dont les activités répondent exactement à notre service social d'aujourd'hui, scientifiquement organisé. La longue expérience de madame Gamelin dans l'exercice des ouvres de charité lui permettait de guider sûrement ses compagnes dans ce pénible et laborieux ministère, tout nouveau pour elles. Avec une maternelle bonté, elle les formait aux diverses tâches d'une Fille de la Charité : visite des malades à domicile; chez les pauvres pour les consoler et les assister; chez les riches pour leur apporter l'aumône de bonnes et encourageantes paroles et faire appel à leur charité en faveur des nécessiteux.

Sœur Gamelin est souvent de la partie, moins souvent que son grand et noble cour l'eût désiré, peut-être, parce qu'elle doit s'occuper de l'administration de l'Asile, recevoir les gens au parloir, intéresser à son œuvre les âmes charitables que la Providence semble se plaire à diriger vers son établissement. Elle reçoit et examine les aspirantes à la vie religieuse, ce qui donne lieu à certaines scènes amusantes et riches d'enseignements comme celle-ci :

Le 26 décembre 1843, Sœur Gamelin, encore novice, est appelée au parloir par M. Joseph Pariseau, menuisier de la paroisse SaintMartin. Il est accompagné de sa fille, Esther, âgée de vingt ans. " Madame », dit-il à Sour Gamelin, " je vous amène ma fille qui veut, à tout prix, se faire religieuse. C'est pour moi une grande perte, mais c'est pour vous une belle acquisition. Ma fille sait lire, écrire, coudre, faire la cuisine, jardiner, etc. Elle peut même travailler le bois et conduire une besogne. Elle vous fera une vraie bonne supérieure, je vous l'assure. \ La jeune fille, confuse, veut protester, mais Sour Gamelin l'interrompt : « Laissez, ma fille, laissez parler votre père; il m'intéresse beaucoup... Malgré la bonne impression qu'elle éprouva de 
cette entrevue, Sœur Gamelin était sans doute loin de penser que, dans la personne de mademoiselle Pariseau, elle recevait au noviciat la femme de génie qui, devenue religieuse sous le nom de Sœur Joseph-du-SacréCœur, devait fonder nos premières et plus belles missions de l'extrême Ouest, canadien et américain

Nous en avons assez dit, semble-t-il, pour que personne ne puisse hésiter à décerner à Mère Gamelin une maîtrise en service social. Si, dans la Communauté, nous avons encore des travailleuses sociales, selon l'espritit de Mère Gamelin nous l'espérons, n'est-ce pas grâce à celle qui leur a tracé un merveilleux chemin, pavé de dévouement, d'abnégation, d'oubli de soi, de tendre pitié pour la misère humaine sous quelque forme qu'elle se présente. Pour la gloire de l'Eglise canadienne et le service des pauvres de Jésus-Christ, puissent-elles garder toujours l'esprit de leur mère Fondatrice, Mère Emilie Gamelin, la première travailleuse sociale de l'Institut de la Providence.

Sœur Madeleine-Durand, f.c.s.p. 